\title{
Distinctive Clinical Features of Mild Cognitive Impairment with Subcortical Cerebrovascular Disease
}

\author{
Samantha Galluzzi ${ }^{\mathrm{a}}$ Ching-Fan Sheu ${ }^{\mathrm{d}}$ Orazio Zanetti ${ }^{\mathrm{b}}$ Giovanni B. Frisoni ${ }^{\mathrm{a}, \mathrm{c}}$ \\ a Laboratory of Epidemiology and Neuroimaging, ${ }^{b}$ Alzheimer's Unit, IRCCS San Giovanni di Dio - FBF, Brescia, \\ and ${ }^{\mathrm{C}} \mathrm{AFaR}$ Associazione Fatebenefratelli per la Ricerca, Roma, Italy; ${ }^{\mathrm{d}}$ Department of Psychology, \\ DePaul University, Chicago, III., USA
}

\section{Key Words}

Mild cognitive impairment $\cdot$ Cerebrovascular disease - Subcortical vascular cognitive impairment . Small vessel disease

\begin{abstract}
Background and Purpose: Patients with mild cognitive impairment and subcortical cerebrovascular disease ( $\mathrm{svMCl}$ ) can be isolated using criteria modified from those of Erkinjuntti et al. for subcortical vascular dementia and have poorer outcomes (cognitive deterioration, disability, institutionalization, and mortality). The aim of this study was to test which of the core (dysexecutive syndrome with relative sparing of memory, gait disorders and extrapyramidal signs) and supporting (urinary and behavioral symptoms) clinical features are most useful to recognize patients with $\mathrm{svMCl}$ and discriminate them from those with amnestic $\mathrm{MCl}(\mathrm{aMCl})$. Methods: Twenty-nine svMCl and $14 \mathrm{aMCl}$ patients were seen in a memory clinic. Tests and scales assessing core and supporting features that independently contributed to the discrimination between $\mathrm{svMCl}$ and $\mathrm{aMCl}$ were identified with stepwise logistic regression analysis. The accuracy of the discrimination was estimated with area under the receiver operating characteristic curve and $95 \%$ confidence intervals (Cls). Results: The most accurate scales
\end{abstract}

were the extrapyramidal sign scale by Richards et al. $(0.75,95 \% \mathrm{Cl} 0.61-0.89)$, letter fluency $(0.75,95 \% \mathrm{Cl} 0.61-$ $0.90)$, irritability of the Neuropsychiatric Inventory and urinary dependence $(0.66,95 \% \mathrm{Cl} 0.49-0.82$ for both), and digit span forward $(0.59,95 \% \mathrm{Cl} 0.41-0.77)$. The overall accuracy of a model compounding information from main and supporting features was $0.98,95 \% \mathrm{Cl} 0.94-1.0$. Conclusions: All the domains that are included in the clinical criteria for svMCl independently contribute to the identification of the condition. These criteria can be useful to recognize svMCl patients in clinical settings.

Copyright (C) 2005 S. Karger AG, Basel

\section{Introduction}

Mild cognitive impairment (MCI) refers to a transitional state between normal aging and dementia, where patients have cognitive impairment at a level not yet fulfilling diagnostic criteria for dementia. MCI is a clinically heterogeneous syndrome comprising a number of conditions [1]. The one relatively more clearly defined is amnestic MCI (aMCI), which refers to individuals with memory deficit and preserved general cognition [2]. Most patients with aMCI have early-stage Alzheimer's disease (AD) [3]. Theoretically, patients with other dementing disorders such as Lewy body dementia, frontotemporal

\section{KARGER \\ Fax +4161306 1234 E-Mail karger@karger.ch} www.karger.com
(C) 2005 S. Karger AG, Basel $1420-8008 / 05 / 0194-0196 \$ 22.00 / 0$

Accessible online at:

www.karger.com/dem
Giovanni B. Frisoni, MD

Laboratory of Epidemiology and Neuroimaging, IRCCS San Giovanni di Dio - FBF via Pilastroni 4, IT-25125 Brescia (Italy)

Tel. +390303501361, Fax +390270043 5727, E-Mail papers@centroAlzheimer.it

http://www.centroAlzheimer.it 
dementia, and vascular dementia might pass through the predementia stage and be captured before the full-blown clinical picture has developed [1]. However, clinical descriptions of patients at this stage are still few and sparse.

Subcortical vascular dementia (SVD) is due to small vessel disease causing lacunar infarcts and other subcortical ischemic changes. SVD patients are a highly prevalent and homogeneous group, with a distinctive clinical picture $[4,5]$. Clinical criteria have recently been proposed to recognize SVD based on imaging and clinical features [6].

The natural history of a group of patients with the predementia stage of SVD [subcortical vascular MCI (svMCI)] has recently been described [7]. This is a highly prevalent condition in clinic. In a series of 501 consecutive patients admitted to our Alzheimer's Unit in a year, it has been found that $14 \%$ had svMCI, while aMCI accounted for $9 \%$ of all patients [5]. When compared to aMCI patients, those with svMCI had a poorer outcome: after a 3-year follow-up, 50\% had died, while all aMCI patients were still alive. Moreover, of those alive, $68 \%$ of svMCI vs. $17 \%$ of aMCI patients were either in a nursing home, or had functional loss or severe cognitive deterioration [7].

The economic impact of this condition is also considerable. Estimates from the Canadian Study of Health and Aging [8] showed that costs for svMCI were much higher than for mild AD (CAD 15,000 vs. 9,500). This difference was mainly due to the institutionalization rate of patients, which was high $(41 \%)$ in vascular demented patients, even if the severity of the dementia was mild. The higher costs associated with vascular dementia suggest that preventive interventions, for example controlling vascular risk factors, might be cost-effective [9].

The clinical criteria used to detect svMCI patients were those of Erkinjuntti et al. [6] for SVD modified to accommodate lower severity of cerebrovascular disease [7]. The criteria require as core features the evidence of relevant cerebrovascular disease by brain imaging, a cognitive profile of a dysexecutive 'frontal' syndrome with relative sparing of memory, and neurological signs (gait disorders and extrapyramidal signs). Supporting features include urinary symptoms such as urgency and incontinence, and behavioral symptoms.

The use of clinical criteria to define a condition implicitly assumes that most or many of the constituting features are contributing to the identification of the disease of interest. The aim of this study was to assess which domains among dysexecutive 'frontal' syndrome, sparing of memory, gait disorders and extrapyramidal signs, urinary symptoms, and behavioral symptoms discriminate svMCI from aMCI patients. The finding that most or all domains are independently necessary would provide indirect validation of the criteria for SVD.

\section{Methods}

\section{Patients}

The patients of this study were recruited in the day hospital section of the Alzheimer's Center of the IRCCS San Giovanni di DioFatebenefratelli, Brescia, Italy. The patients were evaluated as part of a prospective study on the natural history of early cognitive impairment and AD (Mini-Mental State Exam score of 18 or higher; The Mild Alzheimer Project) started on January 1st, 1996 and terminated on December 31st, 2000. After a complete description of the study, written informed consent was obtained from the patients or their primary caregiver. The study was reviewed and approved by the local ethics committee.

\section{Assessment}

The baseline evaluation included a complete sociodemographic and clinical (cognitive, behavioral, neurological, functional, and physical) data collection. History was taken from a knowledgeable informant (usually the patient's spouse) and was focused on those symptoms that might help in the diagnostic differentiation of the most frequent forms of cognitive impairment: implicit and explicit memory, language and executive functions, behavioral disturbances, disability in daily activities, hallucinations and other psychiatric symptoms, and falls. All patients underwent laboratory exams and computed tomography (CT) for the routine workup of cognitive impairment. At the end of the clinical, neuropsychological, and instrumental evaluation, the clinical diagnosis was made by consensus among the physicians-in-charge (a neurologist and a geriatrician), the neuropsychologist, and nurses and occupational therapists who had taken care of or had come in contact with the patient during the stay. A full description of the clinical assessment of the patients of this study is detailed elsewhere [7]. Here we report a description of the variables relevant to this study.

Clinical Diagnoses. The diagnosis of aMCI was made according to the Mayo Clinic criteria [2] and that of svMCI according to a modified version [7] (table 1) of the criteria for SVD by Erkinjuntti et al. [6]. Modifications regarded disability and the severity of CT-assessed lesions and were aimed to include patients whose cognitive syndrome is not interfering with complex occupational and social activities and whose CT scan showed vascular lesions of mild severity. The dysexecutive syndrome and memory deficit were defined on the basis of a clinical neuropsychological assessment and confirmed through testing. Accordingly, 29 patients with svMCI and 14 with aMCI were identified, amounting to 15 and $7 \%$ of all the 195 patients enrolled in the Mild Alzheimer Project.

Clinical Features. Normal general cognitive function was defined as having a Mini-Mental State Examination [10] score of 24 and higher or Clinical Dementia Rating [11] of 0.5. Risk factors were hypertension, diabetes, high serum cholesterol, and past or present smoking. Cardiovascular diseases were: heart diseases of ischemic pathogenesis, primary arrhythmias, and heart failure, as- 
Table 1. Criteria used to define MCI with subcortical vascular features

(I) Main diagnostic features

(A) Cognitive syndrome

(1) Dysexecutive syndrome

Impairment in goal formulation, initiation, planning, organizing, sequencing, executing, set-shifting and self-maintenance, and abstracting

(2) Memory deficit (may be mild)

Impaired recall, relative intact recognition, less severe forgetting, benefit from cues

(3) Progression

Deterioration of dysexecutive syndrome and memory deficit from a previous higher level of functioning that is not per se interfering with complex (executive) occupational and social activities

(B) Cerebrovascular disease

(1) Evidence of relevant cerebrovascular disease by brain imaging defined as the presence of

(a) Extensive periventricular and deep white matter lesions: patchy areas of low attenuation (intermediate density between that of normal white matter and that of intraventricular cerebrospinal fluid) or diffuse symmetrical areas of low attenuation with ill-defined margins extending to the centrum semiovale plus at least one lacunar infarct

(b) Absence of cortical and/or corticosubcortical nonlacunar territorial infarcts and watershed infarcts, hemorrhages indicating large vessel disease, signs of normal pressure hydrocephalus, and specific causes of white matter lesions (e.g. multiple sclerosis, sarcoidosis, brain irradiation)

(2) Presence or a history of neurologic signs

Evidence for cerebrovascular disease such as hemiparesis, lower facial weakness, Babinski sign, sensory deficit, dysarthria, gait disorder, extrapyramidal signs consistent with subcortical brain lesion(s)

(II) Clinical features supporting the diagnosis

(A) Episodes of mild upper motor neuron involvement such as drift, reflex asymmetry, incoordination

(B) Early presence of a gait disturbance (small-step gait or 'marche à petits pas', magnetic, apraxic-ataxic or pakinsonian gait)

(C) History of unsteadiness and frequent, unprovoked falls

(D) Early urinary frequency, urgency, and other urinary symptoms not explained by urologic disease

(E) Dysarthria, dysphagia, extrapyramidal signs (hypokinesia, rigidity)

(F)Behavioral and psychological symptoms such as depression, personality change, emotional incontinence, psychomotor retardation

(III) Features that make the diagnosis uncertain or unlikely

(A) Early onset of memory deficit and progressive worsening of memory and other cognitive functions such as language (transcortical sensory aphasia), motor skills (apraxia), and perception (agnosia), in the absence of corresponding focal lesions on brain imaging

(B) Absence of relevant cerebrovascular lesions on brain CT or MRI

The criteria were modified from Erkinjuntti et al. [6] as described in the text.

certained as described in Frisoni et al. [7]. The Hachinski ischemic score in its original form [12] was computed. The score was categorized into the three levels of $0,1-4$, and 5 or more.

Neuropsychological Assessment. This consisted of a battery of tests tapping frontal [category and letter word fluency [13], Wisconsin Card Sorting Test (WCST) [14] categories, errors, and perseverations] and memory (prose recall [15], digit span forward [16], Corsi test [16]) functions. Verbal fluency and WCST were selected to tap frontal functions because of their large use in clinical practice and well-recognized sensitivity to frontal lobe damage [17, 18]. Except for the WCST, normative reference values for the Italian population are available $[13,15,16]$ and the crude test performance scores can be transformed into age-, gender- and education-corrected scores. The judgement of normality was given based on these norms, allowing to categorize cognitive performance into abnormal (below the 5th percentile of the distribution of the elderly reference population), borderline (5th to 10th percentile), and low normal (10th to 25 th percentile). Lacking Italian population norms, abnormal performance on the WCST was judged by an expert neuropsychologist.

Subcortical Vascular Features. Balance was assessed with the Tinetti scale [19], a performance-based assessment of mobility. The score ranges from 0 to 16 (best performance). Gait was evaluated as the time to walk $15 \mathrm{~m}$, as described by Reuben and Siu [20]. Parkinsonism was assessed with the extrapyramidal sign (EPS) scale by Richards et al. [21]. The EPS scale was divided into two subscales, one comprising balance and gait (score 0-8), and the other comprising all the other disturbances (score 0-20), higher scores indicating greater severity. Urinary dependence was defined as total or partial dependence in urinary function, based on the pertinent item of the Barthel Index [22]. The Neuropsychiatric Inventory [23] was used to assess behavioral disturbances. Only sub- 
scales with a prevalence higher or equal to $20 \%$ were used in the present study (agitation/aggression, depression, anxiety, apathy, and irritability/lability). The theoretical score for each subscale ranges between 0 and 12 (maximum severity).

\section{Statistical Analysis}

The ability of frontal and memory tests, gait disorders and extrapyramidal signs, urinary and behavioral symptoms to discriminate between svMCI and aMCI was evaluated by the c-statistic using SPSS version 10.1. The c-statistic is equal to the area under the receiver operating characteristic (ROC) curve (AUC). This is a frequently used measure of effectiveness of diagnostic tests in discriminating between groups of individuals [24]. The ROC curve plots the true positive rate (i.e. sensitivity) against the false-positive rate (i.e. 1 - specificity) for different cutoffs. The AUC ranges in value from 0.5 (test is no better than chance for assigning cases to outcomes) to 1 (test always correctly classifies cases into outcomes). Therefore, the closer the ROC plot is to the upper left corner, the higher the overall accuracy of the test [25].

To compute the c-statistic, logistic regression was first carried out to calculate the predicted probability of the dichotomous outcome (svMCI or aMCI). Then, using the ROC facility of SPSS, the AUC with 95\% confidence intervals (CIs) was computed. If the CI does not include the 0.5 value (chance value), there is evidence that the test has the ability to discriminate between the two groups [24].

To identify discriminating variables, we performed a series of bivariate logistic regressions to predict the dichotomous outcome (svMCI or aMCI) using individual scales from the domains of neuropsychological tests, gait disorders and extrapyramidal signs, urinary and behavioral symptoms. Then, we performed a series of multiple logistic regressions for each domain on all the scales. Lastly, we sought to construct the best predictive model in two ways: (1) selecting the 'best' predictor from each of the domains according to the maximum c-statistic from the series of simple logistic regressions; (2) using the (automated) stepwise variable selection procedure with significance for entry and exit of $p<0.20$. The choice of this $\mathrm{p}$ level, which is higher than the traditional choices of 0.05 or 0.1 , produces a subset of predictors that is consistent with the one-variable-at-a-time model selection.

\section{Results}

Table 2 shows that patients with svMCI were 6 years older than those with aMCI. Cognitive impairment was similar, while vascular risk factors and cardiovascular diseases were more frequent in svMCI than aMCI patients. Most of svMCI patients had an Hachinski ischemic score of 4 or lower.

The performance on frontal neuropsychological tests was poorer in svMCI than in aMCI patients, with variable degrees of statistical significance, only WCST perseverations failing to achieve statistical significance. On the other hand, performance on all the tests tapping memory was similar in the two groups. Gait and extrapyramidal
Table 2. Clinical features of patients with svMCI and aMCI

\begin{tabular}{lccc}
\hline & $\begin{array}{c}\text { svMCI } \\
(\mathrm{n}=29)\end{array}$ & $\begin{array}{l}\text { aMCI } \\
(\mathrm{n}=14)\end{array}$ & $\mathrm{p}$ \\
& & & \\
Sociodemographics & $78 \pm 7$ & $72 \pm 9$ & 0.04 \\
Age, years & $15(52)$ & $8(57)$ & 0.50 \\
Female & $6 \pm 3$ & $6 \pm 2$ & 0.98 \\
Education, years & & & \\
\hline Cognitive status & & & \\
Mini-Mental State Examination & $24.3 \pm 2.6$ & $24.7 \pm 2.4$ & 0.52 \\
$\quad$ score & & & \\
\hline $\begin{array}{l}\text { Vascular features } \\
\text { Vascular risk factors }\end{array}$ & $3(10)$ & $6(43)$ & 0.05 \\
$\quad 0$ & $17(59)$ & $5(36)$ & \\
$\quad 1$ & $9(31)$ & $3(21)$ & \\
$\quad 2+$ & & & \\
Cardiovascular diseases & $14(48)$ & $11(79)$ & 0.09 \\
$\quad 0$ & $15(52)$ & $3(21)$ & \\
$\quad 1+$ & & & \\
Hachinski ischemic score & $6(43)$ & $2(7)$ & 0.002 \\
$\quad 0$ & $7(50)$ & $16(55)$ & \\
$\quad 1-4$ & $1(7)$ & $11(38)$ & \\
$5+$ & & &
\end{tabular}

$\mathrm{p}$ denotes the significance of the difference between svMCI and aMCI on exact tests: $\chi^{2}$ test (with Fisher's correction when 1 or more cells had $\mathrm{n}<5$ ) for dichotomous variables, Mann-Whitney test for continuous and Kruskal-Wallis test for ordinal variables (Monte Carlo method, sampling $n=10,000$ ). Vascular risk factors: hypertension, diabetes, high serum cholesterol, and past or present smoking; cardiovascular diseases: heart diseases of ischemic pathogenesis, primary arrhythmias, and heart failure. Figures denote numbers, with percentages in parentheses or means \pm SD.

signs were poorer in svMCI than in aMCI patients on the basis of all assessment instruments. None of the aMCI vs. $31 \%$ of svMCI patients had urinary symptoms. Behavioral symptoms were more severe in svMCI (almost 3 out of the 5 symptoms examined), although the differences were not statistically significant (table 3 ).

When the contribution of individual scales from neuropsychological tests, gait disorders and extrapyramidal signs, and behavior was compounded to discriminate the two groups ('all scales for each domain' in table 3), the AUC indicated fair accuracy for frontal tests, gait disorders and extrapyramidal signs, and behavior (0.74-0.79). On the other hand, the accuracy of memory test was poor, as indicated by a low AUC (0.63) with the $95 \%$ CI including the null value $(0.45-0.81)$. The accuracy of urinary symptoms was also relatively low (AUC 0.66), but its $95 \%$ CI was from 0.49 to 0.82 , indicating some ability to discriminate between the two groups. 
Table 3. Separation of svMCI from aMCI based on neuropsychological tests, gait disorders and extrapyramidal signs, urinary and behavioral symptoms

\begin{tabular}{|c|c|c|c|c|}
\hline \multirow[t]{2}{*}{ Domain, scale } & \multicolumn{2}{|c|}{ Scores, means $\pm \mathrm{SD}$} & \multicolumn{2}{|l|}{ Accuracy } \\
\hline & svMCI & aMCI & individual scales & $\begin{array}{l}\text { all scales for } \\
\text { each domain }\end{array}$ \\
\hline \multicolumn{5}{|l|}{ Frontal tests } \\
\hline Letter fluency & $22.5 \pm 9.1$ & $26.3 \pm 5.1^{\mathrm{a}}$ & $0.75(0.61-0.90)$ & \multirow{5}{*}{$0.79(0.64-0.94)$} \\
\hline Category fluency & $27.5 \pm 7.7$ & $31.9 \pm 5.1^{\mathrm{a}}$ & $0.70(0.54-0.85)$ & \\
\hline WCST categories & $1.2 \pm 1.0$ & $1.9 \pm 1.4^{\mathrm{a}}$ & $0.67(0.48-0.86)$ & \\
\hline WCST errors & $25.7 \pm 11.7$ & $21.6 \pm 7.0^{\mathrm{a}}$ & $0.62(0.44-0.80)$ & \\
\hline WCST perseverations & $11.0 \pm 8.9$ & $9.1 \pm 4.7$ & $0.52(0.33-0.70)$ & \\
\hline \multicolumn{5}{|l|}{ Memory tests } \\
\hline Babcock & $6.8 \pm 4.1$ & $5.8 \pm 4.5$ & $0.58(0.39-0.77)$ & \multirow{3}{*}{$0.63(0.45-0.81)$} \\
\hline Digit span forward & $5.5 \pm 1.0$ & $5.3 \pm 0.6$ & $0.59(0.41-0.77)$ & \\
\hline Corsi & $4.0 \pm 0.9$ & $4.1 \pm 0.8$ & $0.52(0.33-0.70)$ & \\
\hline \multicolumn{5}{|c|}{ Gait disorders and extrapyramidal signs } \\
\hline EPS stance and gait & $1.1 \pm 1.1$ & $0.1 \pm 0.4^{\mathrm{a}}$ & $0.75(0.61-0.89)$ & \multirow{4}{*}{$0.79(0.65-0.93)$} \\
\hline EPS other symptoms & $2.4 \pm 2.6$ & $0.7 \pm 1.7^{\mathrm{a}}$ & $0.75(0.59-0.91)$ & \\
\hline Balance (Tinetti scale) & $12.0 \pm 3.0$ & $14.0 \pm 1.2^{\mathrm{a}}$ & $0.70(0.55-0.86)$ & \\
\hline Time to walk $15 \mathrm{~m} / \mathrm{s}$ & $20.5 \pm 12.3$ & $4.4 \pm 3.4^{\mathrm{a}}$ & $0.73(0.57-0.89)$ & \\
\hline \multicolumn{5}{|l|}{ Urinary symptoms } \\
\hline Urinary dependence & $9(31 \%)$ & $0^{\mathrm{a}}$ & $0.66(0.49-0.82)$ & \\
\hline \multicolumn{5}{|l|}{ Behavior } \\
\hline Agitation/aggression & $1.9 \pm 3.3$ & $1.5 \pm 2.8$ & $0.53(0.34-0.71)$ & \multirow{5}{*}{$0.74(0.59-0.90)$} \\
\hline Depression & $2.5 \pm 4.0$ & $3.1 \pm 3.9$ & $0.57(0.38-0.75)$ & \\
\hline Anxiety & $2.3 \pm 3.7$ & $1.5 \pm 2.4$ & $0.54(0.36-0.72)$ & \\
\hline Apathy & $3.6 \pm 4.5$ & $3.6 \pm 4.2$ & $0.53(0.35-0.71)$ & \\
\hline Irritability/lability & $2.5 \pm 3.4$ & $0.6 \pm 1.7$ & $0.66(0.49-0.82)$ & \\
\hline
\end{tabular}

Accuracy denotes the AUC (95\% CIs). Urinary symptoms were assessed with the continence item from the Barthel Index; behavior was assessed with the Neuropsychiatry Inventory.

${ }^{a}$ Significance of the difference $(\mathrm{p}<0.05)$ between svMCI and aMCI on exact tests: $\chi^{2}$ test (with Fisher's correction when 1 or more cells had $\mathrm{n}<5$ ) for dichotomous, and Mann-Whitney test for continuous variables.

Letter fluency, digit span forward, ESP stance and gait, and irritability were the best predictors from each of the domains of neuropsychological tests, gait disorders and extrapyramidal signs, and behavior, based on the AUC value. These, together with urinary dependence, were included in a multiple logistic regression that yielded an AUC value of 0.84 (95\% CI: 0.71-0.97) (fig. 1). A logistic regression model where all variables of table 2 were tested for inclusion with an automated stepwise variable selection procedure selected as best predictors the WCST error test, Corsi test, EPS stance and gait, urinary dependence and agitation. The resulting AUC was excellent $(0.98,95 \%$ CI: 0.94-1.0) (fig. 1). It should be noted that this algorithm of variable selection did not have any domain information, and - statistically - it might have selected one or more scales from the same domain provided they increased group separation.

\section{Discussion}

We have found that all the main and supporting features identified a priori by Erkinjuntti et al. [6] for SVD independently contribute to separate svMCI from aMCI patients. A corollary of these findings is that criteria for svMCI [7] are valid, i.e. they have internal consistency.

Although still debated, increasing evidence supports the existence of svMCI. Meyer et al. [26] in an 8-year longitudinal study of 291 cognitively normal subjects found that 27 developed vascular dementia. Of these, 15 $(56 \%)$ developed vascular dementia from prodromal MCI stage and 12 (44\%) directly from normal cognitive status, without passing through the MCI stage. The comparison of clinical and neuroradiological characteristics between the two groups showed that most vascular demented patients with prodromal MCI had SVD, while 


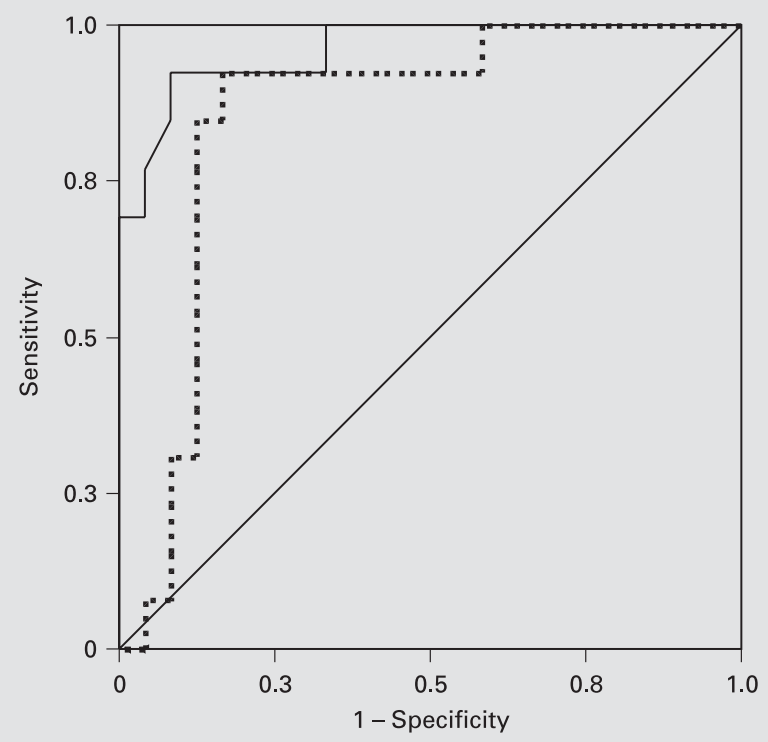

Fig. 1. Accuracy of discrimination between svMCI and aMCI. ROC curve for the best discriminant scales for each domain $(\mathrm{AUC}=0.84$, 95\% CI 0.71-0.97) and for scales selected by stepwise logistic regression (AUC $=0.98,95 \%$ CI 0.94-1.0). Dotted line: based on a logistic model where the best discriminating scales of each of the 5 domains were entered (letter fluency, digit span forward, EPS stance and gait, urinary dependence and irritability). Solid line: based on an algorithmic (stepwise) procedure for variable selection. The algorithm selected one scale for each domain: WCST errors for frontal tests, Corsi for memory tests, EPS stance and gait for gait disorders, urinary dependence for urinary symptoms, and agitation for behavior.

most of those without prodromal MCI had multi- or strategic infarct dementia. Traykov et al. [27] found that apolipoprotein e4 allele was associated with aMCI and $\mathrm{AD}$, but not with vascular MCI and SVD, suggesting that vascular MCI is separate from aMCI. Pugh and Lipsitz [28] collected pathophysiological, epidemiological, and clinical evidence from the literature supporting the notion of a 'frontal-subcortical syndrome of aging' related to subcortical cerebrovascular disease. This syndrome would include as main domains a cognitive syndrome (executive dysfunction), gait abnormalities (parkinsonism), mood disorders, and urinary dysfunction (urgency/incontinence).

Although we cannot exclude that our svMCI patients would show $\mathrm{AD}$ on pathology, the clinical features that we found characterizing these patients are quite inconsistent with what is known of the pathophysiology and nat- ural history of AD. Gait disturbance at the onset or very early in the course of dementia is not typical for $\mathrm{AD}$ [29]. A clinical-neuropathologic study showed that none of the 36 patients with mild dementia from a series of 95 autopsy-proven AD patients had abnormal gait [30]. In a prospective community study of 422 nondemented elderly followed for a median of 7 years, subjects with abnormal gait had greater risk of developing vascular dementia (hazard ratio 3.5), but not $\mathrm{AD}$ [31]. In a subgroup of 48 patients where autopsy was performed, vascular dementia, in particular micro-infarcts, was the pathological condition most common in subjects with abnormal gait [31].

Urinary symptoms such as urgency and incontinence are typically present in the more advanced stages of $\mathrm{AD}$ [32]. On the contrary, incontinence is a frequent and early manifestation of SVD [33], has been described in nondemented subjects with leukoaraiosis [34], and might precede cognitive impairment by more than 5 years [35].

A distinctive profile of behavioral symptoms for different forms of dementia, such as SVD and AD, has yet to be defined. A population study of 362 patients with dementia and 320 with MCI confirmed the high prevalence of behavioral symptoms in dementia, and a moderate prevalence in MCI [36]. In fact, $80 \%$ of demented and $50 \%$ of MCI patients showed at least one symptom, evaluated with the Neuropsychiatry Inventory, from the onset of cognitive impairment. The most frequent clinically significant symptoms in MCI were sleep disturbances and irritability. However, MCI patients were not further categorized into amnestic and vascular groups [36].

The neuropsychological profile we found in svMCI patients compared to aMCI is that of impairment of frontal functions, with no substantial differences in memory. Several studies have found frontal executive dysfunction in SVD, supporting its relevance in the differential diagnosis of AD [37-40]. Some have even shown better recognition memory in SVD, while delayed recall was equally impaired, perhaps reflecting retrieval deficit [38, 40]. However, the lack of pathological studies in SVD and svMCI patients who also had accurate neuropsychological testing prevents confirmation of this view.

The contribution of vascular and degenerative lesions to cognitive impairment in subcortical cerebrovascular disease remains controversial. Imaging studies have shown that dementia in subcortical cerebrovascular disease correlates with hippocampal and cortical atrophy, rather than with any measure of lacunar damage [41, 42]. However, grey matter atrophy does not point per se to neurodegeneration as it can also result from white matter 
vascular pathology [43]. Results from 4 autopsied cases with subcortical cerebrovascular disease and hippocam$\mathrm{pal} /$ cortical atrophy showed primary pathologic diagnosis of ischemic vascular disease, in the absence of AD pathology in the neocortex [41]. A prospective clinicopathological study of 28 demented patients showed that the severity of cognitive impairment significantly correlated with the volume of infarcts and lacunes, but not with the density of neuritic plaques and neurofibrillary tangles [44].

Some methodological issues deserve clarification. It might be argued that MCI diagnoses are based on criteria and the scales included in them are used to test the accuracy of these criteria, representing circular reasoning. We believe that this is not the case for at least two reasons. First, the diagnostic criteria for SVD and svMCI were originally developed based on subjective clinical perception, rather than derived from empirical data. It is therefore necessary to test how subjective perception corresponds to empirical data. Second, even when assuming that the criteria of Erkinjuntti et al. [6] are valid, it does not predict which scales or domains or combination of scales or domains will yield good accuracy figures. This is due to the fact that the application of clinical criteria in the diagnostic workup of our patients followed - as is always the case in the clinical routine - a holistic rather than algorithmic approach.

The same question applies to neuroimaging: how much does this contribute - if any - to separate groups? The presence of neuroimaging among the domains of the diagnostic criteria does not per se imply that it provides incremental diagnostic information in addition to that provided by the clinical variables. This will need to be addressed in future studies.

svMCI is a prevalent condition with a definite clinical, neuropathological, and behavioral profile. The high risk of adverse outcomes makes the condition a target for therapeutic interventions. The availability of valid diagnostic criteria is preliminary to the setting of intervention trials and devising appropriate strategies that may help slowing or halting the progression of the disease.

\section{References}

- 1 Petersen RC, Doody R, Kurz A, Mohs RC, Morris JC, Rabins PV, Ritchie K, Rossor M, Thal L, Winblad B: Current concepts in mild cognitive impairment. Arch Neurol 2001;58: 1985-1992.

>2 Petersen RC, Smith GE, Waring SC, Ivnik RJ, Tangalos EG, Kokmen E: Mild cognitive impairment: Clinical characterization and outcome. Arch Neurol 1999;56:303-308.

\3 Morris JC, Storandt M, Miller JP, McKeel DW, Price JL, Rubin EH, Berg L: Mild cognitive impairment represents early-stage Alzheimer disease. Arch Neurol 2001;58:397405.

-4 Erkinjuntti T, Inzitari D, Pantoni L, Wallin A, Scheltens P, Rockwood K, Desmond DW: Limitations of clinical criteria for the diagnosis of vascular dementia in clinical trials. Is a focus on subcortical vascular dementia a solution? Ann NY Acad Sci 2000;903:262-272.

5 Galluzzi S, Cimaschi L, Ferrucci L, Frisoni GB: Mild cognitive impairment: Clinical features and review of screening instruments. Aging (Milano) 2001;13:183-202.

-6 Erkinjuntti T, Inzitari D, Pantoni L, Wallin A, Scheltens P, Rockwood K, Roman GC, Chui H, Desmond DW: Research criteria for subcortical vascular dementia in clinical trials. J Neural Transm Suppl 2000;59:23-30.

>7 Frisoni GB, Galluzzi S, Bresciani L, Zanetti O, Geroldi C: Mild cognitive impairment with subcortical vascular features: Clinical characteristics and outcome. J Neurol 2002;249: 1423-1432.
8 Rockwood K, Brown M, Merry H, Sketris I, Fisk J: Vascular Cognitive Impairment Investigators of the Canadian Study of Health and Aging: Societal costs of vascular cognitive impairment in older adults. Stroke 2002;33. 1605-1609.

9 Fillit H, Hill J: The costs of vascular dementia. A comparison with Alzheimer's disease. J Neurol Sci 2002;203-204:35-39.

10 Folstein MF, Folstein SE, McHugh PR: 'MiniMental State'. A practical method for grading the cognitive state of patients for the clinician. J Psychiatr Res 1975;12:189-198.

11 Morris JC: The Clinical Dementia Rating (CDR): Current version and scoring rules. Neurology 1993;43:2412-2414.

12 Hachinski VC, Iliff LD, Zilhka E, Du Boulay GH, McAllister VL, Marshall J, Russell RW, Symon L: Cerebral blood flow in dementia. Arch Neurol 1975;32:632-637.

13 Novelli G, Papagno C, Capitani E, Laiacona M, Vallar G, Cappa SF: Tre test clinici di ricerca e produzione lessicale. Taratura su soggetti normali. Arch Psicol Neurol Psichiatr 1986;47:477-505.

14 Heaton RK: Wisconsin Card Sorting Test Manual. Odessa, Psychological Assessment Resources Inc, 1981.

15 Spinnler H, Tognoni G: Standardizzazione e taratura italiana di test neuropsicologici. Ital J Neurol Sci 1987;6(suppl 8):1-120.
16 Orsini A, Grossi D, Capitani E, Laiacona M, Papagno C, Vallar G: Verbal and spatial immediate memory span: Normative data from 1,355 adults and 1,112 children. Ital J Neurol Sci 1987;8:539-548.

17 Demakis GJ: A meta-analytic review of the sensitivity of the Wisconsin Card Sorting Test to frontal and lateralized frontal brain damage. Neuropsychology 2003; 17:255-264.

18 Hodges JR: Frontotemporal dementia (Pick's disease): Clinical features and assessment. Neurology 2001;56(suppl 4):S6-S10.

$>19$ Tinetti ME: Performance-oriented assessment of mobility problems in elderly patients. J Am Geriatr Soc 1986;34:119-126.

20 Reuben DB, Siu AL: An objective measure of physical function of elderly outpatients. The Physical Performance Test. J Am Geriatr Soc 1990;38:1105-1112.

-21 Richards M, Marder K, Bell K, Dooneief G, Mayeux R, Stern Y: Interrater reliability of extrapyramidal signs in a group assessed for dementia. Arch Neurol 1991;48:1147-1149.

-22 Mahoney FI, Barthel DW: Functional evaluation: The Barthel Index. Md State Med J 1965; 14:61-65.

-23 Cummings JL, Mega M, Gray K, RosenbergThompson S, Carusi DA, Gornbein J: The Neuropsychiatric Inventory: Comprehensive assessment of psychopathology in dementia. Neurology 1994;44:2308-2314. 
-24 Hanley JA, McNeil BJ: The meaning and use of the area under a receiver operating characteristic (ROC) curve. Radiology 1982;143:2936.

-25 Zweig MH, Campbell G: Receiver-operating characteristic (ROC) plots: A fundamental evaluation tool in clinical medicine. Clin Chem 1993;39:561-577.

-26 Meyer JS, Xu G, Thornby J, Chowdhury MH, Quach M: Is mild cognitive impairment prodromal for vascular dementia like Alzheimer's disease? Stroke 2002;33:1981-1985.

27 Traykov L, Rigaud AS, Baudic S, Smagghe A, Boller F, Forette F: Apolipoprotein E varepsilon 4 allele frequency in demented and cognitively impaired patients with and without cerebrovascular disease. J Neurol Sci 2002; 203-204:177-181.

-28 Pugh KG, Lipsitz LA: The microvascular frontal-subcortical syndrome of aging. Neurobiol Aging 2002;23:421-431.

-29 McKhann G, Drachman D, Folstein M, Katzman R, Price D, Stadlan EM: Clinical diagnosis of Alzheimer's disease: Report of the NINCDS-ADRDA Work Group under the auspices of Department of Health and Human Services Task Force on Alzheimer's Disease. Neurology 1984;34:939-944.

-30 Ala TA, Frey WH 2nd: Validation of the NINCDS-ADRDA criteria regarding gait in the clinical diagnosis of Alzheimer disease. A clinicopathologic study. Alzheimer Dis Assoc Disord 1995;9:152-159.
31 Verghese J, Lipton RB, Hall CB, Kuslansky G, Katz MJ, Buschke H: Abnormality of gait as a predictor of non-Alzheimer's dementia. N Engl J Med 2002;347:1761-1768.

32 Honig LS, Mayeux R: Natural history of Alzheimer's disease. Aging (Milano) 2001;13: 171-182.

33 Cummings JL: Vascular subcortical dementias: clinical aspects. Dementia 1994;5:177180.

-34 Sakakibara R, Hattori T, Uchiyama T, Yamanishi T: Urinary function in elderly people with and without leukoaraiosis: Relation to cognitive and gait function. J Neurol Neurosurg Psychiatry 1999;67:658-660.

35 Kotsoris H, Barclay LL, Kheyfets S, Hulyalkar A, Dougherty J: Urinary and gait disturbances as markers for early multi-infarct dementia. Stroke 1987;18:138-141.

36 Lyketsos CG, Lopez O, Jones B, Fitzpatrick AL, Breitner J, DeKosky S: Prevalence of neuropsychiatric symptoms in dementia and mild cognitive impairment: Results from the cardiovascular health study. JAMA 2002;288:14751483.

37 Graham NL, Emery T, Hodges JR: Distinctive cognitive profiles in Alzheimer's disease and subcortical vascular dementia. J Neurol Neurosurg Psychiatry 2004;75:61-71.

38 Lafosse JM, Reed BR, Mungas D, Sterling SB, Wahbeh H, Jagust WJ: Fluency and memory differences between ischemic vascular dementia and Alzheimer's disease. Neuropsychology 1997;11:514-522.
39 Looi JC, Sachdev PS: Differentiation of vascular dementia from AD on neuropsychological tests. Neurology 1999;53:670-678.

40 Tierney MC, Black SE, Szalai JP, Snow WG, Fisher RH, Nadon G, Chui HC: Recognition memory and verbal fluency differentiate probable Alzheimer disease from subcortical ischemic vascular dementia. Arch Neurol 2001;58: 1654-1659.

41 Fein G, Di Sclafani V, Tanabe J, Cardenas V, Weiner MW, Jagust WJ, Reed BR, Norman D, Schuff N, Kusdra L, Greenfield T, Chui H: Hippocampal and cortical atrophy predict dementia in subcortical ischemic vascular disease. Neurology 2000;55:1626-1635.

42 Mungas D, Jagust WJ, Reed BR, Kramer JH, Weiner MW, Schuff N, Norman D, Mack WJ, Willis L, Chui HC: MRI predictors of cognition in subcortical ischemic vascular disease and Alzheimer's disease. Neurology 2001;57: 2229-2235.

43 Du AT, Schuff N, Laakso MP, Zhu XP, Jagust WJ, Yaffe K, Kramer JH, Miller BL, Reed BR, Norman D, Chui HC, Weiner MW: Effects of subcortical ischemic vascular dementia and $\mathrm{AD}$ on entorhinal cortex and hippocampus. Neurology 2002;58:1635-1641.

$\checkmark 44$ Zekry D, Duyckaerts C, Moulias R, Belmin J, Geoffre C, Herrmann F, Hauw JJ: Degenerative and vascular lesions of the brain have synergistic effects in dementia of the elderly. Acta Neuropathol (Berl) 2002;103:481-487. 